\title{
A microcosting study of microsurgery, LINAC radiosurgery, and gamma knife radiosurgery in meningioma patients
}

\author{
Siok Swan Tan • Erik van Putten • Wideke M. Nijdam • \\ Patrick Hanssens • Guus N. Beute • Peter J. Nowak • \\ Clemens M. Dirven • Leona Hakkaart-van Roijen
}

Received: 5 February 2010/Accepted: 16 May 2010/Published online: 6 June 2010

(c) The Author(s) 2010. This article is published with open access at Springerlink.com

\begin{abstract}
The aim of the present study is to determine and compare initial treatment costs of microsurgery, linear accelerator (LINAC) radiosurgery, and gamma knife radiosurgery in meningioma patients. Additionally, the followup costs in the first year after initial treatment were assessed. Cost analyses were performed at two neurosurgical departments in The Netherlands from the healthcare providers' perspective. A total of 59 patients were included, of whom 18 underwent microsurgery, 15 underwent LINAC radiosurgery, and 26 underwent gamma knife radiosurgery. A standardized microcosting methodology was employed to ensure that the identified cost differences would reflect only actual cost differences. Initial treatment costs, using equipment costs per fraction, were $€ 12,288$ for microsurgery, $€ 1,547$ for LINAC radiosurgery, and $€ 2,412$ for gamma knife radiosurgery. Higher initial treatment costs for microsurgery were predominantly due to inpatient stay $(€ 5,321)$ and indirect costs $(€ 4,350)$. LINAC and
\end{abstract}

S. S. Tan $(\bowtie) \cdot$ L. Hakkaart-van Roijen

Erasmus Universiteit Rotterdam, Institute for Medical

Technology Assessment, P.O. Box 1738, 3000 DR Rotterdam,

The Netherlands

e-mail: tan@bmg.eur.nl

E. van Putten - C. M. Dirven

Department of Neurosurgery, Erasmus MC University Medical Center, P.O. Box 2040, 3000 CA Rotterdam, The Netherlands

W. M. Nijdam · P. J. Nowak

Department of Radiation Oncology, Erasmus MC University

Medical Center, P.O. Box 2040, 3000 CA Rotterdam,

The Netherlands

P. Hanssens - G. N. Beute

St. Elisabeth Hospital, Gamma Knife Center, P.O. Box 90151, 5000 LC Tilburg, The Netherlands gamma knife radiosurgery were equally expensive when equipment was valued per treatment $(€ 2,198$ and $€ 2,412$, respectively). Follow-up costs were slightly, but not significantly, higher for microsurgery compared with LINAC and gamma knife radiosurgery. Even though initial treatment costs were over five times higher for microsurgery compared with both radiosurgical treatments, our study gives indications that the relative cost difference may decrease when follow-up costs occurring during the first year after initial treatment are incorporated. This reinforces the need to consider follow-up costs after initial treatment when examining the relative costs of alternative treatments.

Keywords Cost comparison - Meningioma ·

Microcosting - Microsurgery · Radiosurgery

\section{Introduction}

Meningiomas are common tumors of the central nervous system that originate from the meningeal coverings of the spinal cord and the brain; they account for up to $30 \%$ of all primary brain tumors [1]. Intracranial meningiomas are most commonly reported in adults in their fourth through sixth decades of life and are more common in women, with a female preponderance of about 2:1 [2]. The incidence of meningiomas is climbing, which may indicate more sensitive diagnostic modalities or increased exposure to environmental risk factors [3]. Meningiomas are generally slow-growing benign tumors with a broad spectrum of clinical characteristics. According to the World Health Organization (WHO) classification, the most commonly used grading system for meningiomas, there are three malignancy grades: benign (grade I), atypical (grade II), and anaplastic or malignant (grade III) [4]. 
The gold-standard procedure for treatment of meningioma is microsurgery. Even though advances in microsurgical approaches have greatly improved patient outcomes for meningioma once thought to be unresectable, long-term cure remains a desirable but elusive goal [5]. Besides, microsurgery is costly, being a labor-intensive inpatient procedure [6-8]. Therefore, stereotactic radiosurgery, the delivery of a high, single dose of radiation to a discrete tumor volume, is increasingly accepted as an alternative to conventional microsurgery in selected patients [8, 9].

Radiosurgery, either with linear accelerator (LINAC) or gamma knife, is able to target surgically inaccessible or difficult lesions and has decreased risk of complications related to surgery and anesthesia [5, 7, 10, 11]. Radiosurgical patients may experience higher quality of life than microsurgical patients, especially in the short term, because radiosurgery is a minimally invasive outpatient procedure $[6,12,13]$. Advantages of LINAC radiosurgery include relatively low costs and high accessibility. Gamma knife radiosurgery has been suggested to be more accurate, but its costs are higher and accessibility lower than LINAC radiosurgery, particularly due to its high equipment costs $[10,14]$.

To date, few studies have compared treatment costs of alternative treatments in patients with intracranial tumor, such as acoustic neuromas and vestibular schwannomas, and most of them are outdated [15-19]. Of the studies performed in the past 5 years, three compared microsurgery with gamma knife radiosurgery [6, 8, 12]. Another study made a cost comparison between LINAC and gamma knife radiosurgery [10]. However, these studies neither compared the initial treatment costs of microsurgery, LINAC radiosurgery, and gamma knife radiosurgery nor compared alternative treatments in meningioma. Therefore, the aim of the present study is to calculate initial treatment costs of microsurgery, LINAC radiosurgery, and gamma knife radiosurgery in meningioma patients from a healthcare providers' perspective.

The microcosting methodology is generally considered to be the gold-standard methodology for costing hospital services, because it identifies all relevant cost components at the most detailed level [20, 21]. The methodology allows for identification of costs per patient and for insight into patient subgroups that might account for a large share of total costs. Therefore, the microcosting methodology was used to calculate initial treatment costs in our meningioma patients.

The healthcare providers' perspective also includes follow-up costs which occur after initial treatment. Ignoring these follow-up costs could result in unfair conclusions regarding the relative costs of alternative treatments $[6,20]$. Therefore, this study additionally aims to calculate the follow-up costs in the first year after initial treatment with microsurgery, LINAC radiosurgery, and gamma knife radiosurgery.

\section{Methods}

To be able to make a truthful comparison between microsurgery, LINAC radiosurgery, and gamma knife radiosurgery patients, only patients with radiologically confirmed benign (WHO grade I) meningioma of diameter $\leq 3.0 \mathrm{~cm}$ were recruited. To ensure strict comparability between treatment groups, microsurgery patients were excluded when they could not have been treated with radiosurgery instead (e.g., abutting optic structures or VIII nerve). In addition, atypical (WHO grade II) and anaplastic or malignant (WHO grade III) patients, patients with meningioma diameter $>3.0 \mathrm{~cm}$, patients receiving fractionated radiotherapy, and patients for whom follow-up cost data was unavailable were excluded from this study. Recruitment took place at the Department of Neurosurgery of the Erasmus MC University Medical Center in Rotterdam (microsurgery and LINAC radiosurgery) and at the Department of Neurosurgery of the St. Elisabeth Hospital in Tilburg (gamma knife radiosurgery). Enrolment took place retrospectively in September 2008. All costs are based on Euro 2007 cost data. Where necessary, costs were adjusted to 2007 using the general price index from the Dutch Central Bureau of Statistics [22].

\section{The microcosting study}

Initial treatment costs of microsurgery, LINAC radiosurgery, and gamma knife radiosurgery were based on a detailed microcosting study, in which resource use and unit costs of direct and indirect cost components were identified. Direct cost components involved diagnostic procedures (medical imaging and laboratory services), consumables (medications and disposables), inpatient stay (at the normal ward and intensive care unit), and labor (including neurosurgeons, anesthesiologists, radiation oncologists, residents, physicists, radiation technicians, operation assistants, and nurses). In The Netherlands, the hospital rather than the government owns its exploited equipment. In agreement with the healthcare providers' perspective, our study therefore additionally considered equipment. Indirect cost components concerned overheads (general expenses, administration and registration, energy, maintenance, insurance, and personnel costs of nonpatient services such as management and administration) and capital (depreciation of buildings and inventory, and interest).

Resource use of diagnostic procedures, consumables, inpatient stay, and equipment (number of treatments per year) was available per individual patient, acquired from 
hospital information systems (bottom-up microcosting). Resource use of labor was collected by means of standardized reporting templates; two medical specialists per hospital department were asked to estimate resource use of labor of an average meningioma patient matching the inclusion criteria.

Unit costs of diagnostic procedures and medications were taken from (financial) hospital databases. Annual costs of disposables were obtained from hospital information systems and divided by the annual number of inpatient days to calculate unit costs per inpatient day. Resource use of inpatient stay was valued using reference unit prices [23]. Unit costs of labor were based on standardized costs per minute, which equaled the normative income divided by the number of workable minutes per year. Normative incomes were based on collective labor agreements. Unit costs of health care utilization are presented in Table 1.

Equipment was valued using replacement and maintenance costs with a discount rate of $4 \%$ and anticipated life expectancy of 10 years, as prescribed by the Dutch Health Authority. The LINAC considered in our study was used for fractionated (multiple fraction) as well as stereotactic (single-fraction) treatments, whereas the gamma knife only performed stereotactic treatments. To be able to determine the influence of alternative calculation methods, equipment costs were determined both per fraction and per treatment. As patients receiving fractionated treatments were excluded from this study, the alternative calculation methods were expected to have a substantial impact on the results.

Annual overhead and capital costs were taken from the annual accounts 2006 and divided by direct costs. Thus, indirect costs were allocated to patients using a marginal mark-up percentage.

Table 1 Unit costs of health care utilization, initial treatment $(€ 2007)$

\begin{tabular}{lr}
\hline Medical imaging services & \\
Computed tomography & 207.50 \\
Magnetic resonance imaging & 269.90 \\
Inpatient stay & 386.28 \\
Inpatient hospital day & \\
Labor (per minute) & 1.46 \\
Medical specialist & 0.56 \\
Resident & 0.56 \\
Laboratory technician & 0.50 \\
Operation assistant & 0.43 \\
Nurse & \\
Equipment (replacement costs) & $3,000,000.00$ \\
Gamma knife & $2,500,000.00$ \\
LINAC
\end{tabular}

Follow-up costs

Follow-up costs included visits to healthcare providers (including the general practitioner, medical specialist, physiotherapist, social worker, and company physician), medical imaging services, inpatient stay, medications, and medical aids (such as wheelchairs, rolling walkers, and walking canes). Follow-up costs involved all resource use occurring during the first year after treatment, including resource use which was unrelated to the meningioma treatment.

Resource use of medical imaging services was based on an established protocol, prescribing one magnetic resonance imaging (MRI) at 13 weeks and one at 52 weeks after initial treatment. Other resource use was obtained from standardized questionnaires which were sent to the home addresses of the recruited patients 4,26 or 52 weeks after initial treatment. The recall period was 4 weeks. Annual follow-up resource use and costs were determined by adding up the values per recall period. The values for the time between the measurement periods (week 5-22 and week 27-48) were established through linear interpolation. However, resource use of medications was not linearly interpolated when interpolation would lead to unrealistic regimens. For example, patients may receive flucloxacillin during the 4-week recall period. As flucloxacillin is generally given during a course of 5-10 days, its costs would be included in the calculations but not linearly interpolated.

Resource use of visits to healthcare providers and inpatient stay was valued using reference unit prices [23]. Unit costs of diagnostic procedures, medications, and medical aids were taken from (financial) hospital databases. Because patients were asked whether they made use of medical aids at every measurement time point, we assumed a once-only purchase with life expectancy of 5 years. Unit costs of health care utilization are presented in Table 2.

Table 2 Unit costs of health care utilization, follow-up (€2007)

\begin{tabular}{lr}
\hline General practitioner (one visit) & 21.35 \\
Medical specialist (one visit) & 59.20 \\
Physiotherapist (one visit) & 24.05 \\
Social worker (one visit) & 24.05 \\
Company physician (one visit) & 59.20 \\
Magnetic resonance imaging & 269.90 \\
Inpatient hospital day & 386.28 \\
Walking cane & 10.00 \\
Rolling walkers & 125.00 \\
Wheelchair & 200.00 \\
DAISY player & 400.00 \\
\hline
\end{tabular}

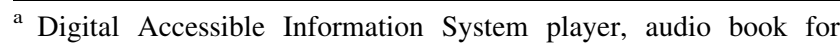
visually impaired patients 
Statistical analyses were conducted using the statistical software program SPSS version 16.0 for Windows. In addition to descriptive statistics, differences between treatment groups and between follow-up scores were assessed by means of one-way analysis of variance (ANOVA) test for variables showing a normal distribution, Kruskal-Wallis test for variables not normally distributed, and Pearson chi-square test for variable fractions. To adjust for multiple testing, ANOVA with post hoc (Bonferroni) testing was additionally performed.

\section{Results}

A total of 143 benign meningioma patients were eligible for this study at our two hospital sites between August 2007 and August 2008. These included 5 atypical (WHO grade II) patients, 31 patients with meningioma diameter
$>3.0 \mathrm{~cm}, 34$ patients receiving fractionated radiotherapy, and 14 patients whose follow-up cost data were unavailable. Thus, a total of 59 patients were recruited, of whom 18 underwent microsurgery, 15 underwent LINAC radiosurgery, and 26 underwent gamma knife radiosurgery. Specific attention was paid to selecting microsurgery patients who could have been treated with radiosurgery instead, thereby preventing important bias.

Table 3 presents the general characteristics at baseline of the patients. LINAC radiosurgery patients reported lower current health state (measured on visual analog scale, with 0 being worst imaginable health and 100 being best imaginable health) compared with microsurgery and gamma knife radiosurgery patients. Tumor volume of microsurgery patients was significantly higher than that of LINAC and gamma knife radiosurgery patients (ANOVA test: $P<$ 0.001 ). No significant differences between treatment groups were observed in any of the other general characteristics.
Table 3 General characteristics of the patients at baseline

\begin{tabular}{|c|c|c|c|}
\hline & Microsurgery & $\begin{array}{l}\text { LINAC } \\
\text { radiosurgery }\end{array}$ & $\begin{array}{l}\text { Gamma knife } \\
\text { radiosurgery }\end{array}$ \\
\hline$n$ & 18 & 15 & 26 \\
\hline \multicolumn{4}{|l|}{ Age } \\
\hline$<31$ years & $0 \%$ & $7 \%$ & $0 \%$ \\
\hline $31-50$ years & $44 \%$ & $20 \%$ & $35 \%$ \\
\hline $51-70$ years & $44 \%$ & $67 \%$ & $42 \%$ \\
\hline$>70$ years & $11 \%$ & $7 \%$ & $23 \%$ \\
\hline \multicolumn{4}{|l|}{ Sex } \\
\hline Male & $16.7 \%$ & $26.7 \%$ & $11.5 \%$ \\
\hline Female & $83.3 \%$ & $73.3 \%$ & $88.5 \%$ \\
\hline Current health state $^{\mathrm{a}}$ & $75.0(\mathrm{SD} 5.8)$ & $66.7(\mathrm{SD} 15.3)$ & $80.0(\mathrm{SD} 11.1)$ \\
\hline \multicolumn{4}{|l|}{ Location of meningioma } \\
\hline Parasagittal & $11 \%$ & $33 \%$ & $0 \%$ \\
\hline Convexity & $11 \%$ & $33 \%$ & $15 \%$ \\
\hline Tuberculum sellae & $28 \%$ & $0 \%$ & $0 \%$ \\
\hline Sphenoid ridge & $22 \%$ & $0 \%$ & $0 \%$ \\
\hline Olfactory groove & $11 \%$ & $13 \%$ & $0 \%$ \\
\hline Falcine, tentorial, and petroclival & $17 \%$ & $20 \%$ & $42 \%$ \\
\hline Cavernous sinus & $0 \%$ & $0 \%$ & $15 \%$ \\
\hline Cerebellopontine angle & $0 \%$ & $0 \%$ & $27 \%$ \\
\hline \multicolumn{4}{|l|}{ Tumor volume } \\
\hline$<5 \mathrm{~cm}^{3}$ & $6 \%$ & $60 \%$ & $46 \%$ \\
\hline $6-10 \mathrm{~cm}^{3}$ & $6 \%$ & $33 \%$ & $42 \%$ \\
\hline $11-15 \mathrm{~cm}^{3}$ & $35 \%$ & $7 \%$ & $8 \%$ \\
\hline $16-20 \mathrm{~cm}^{3}$ & $24 \%$ & $0 \%$ & $0 \%$ \\
\hline $21-25 \mathrm{~cm}^{3}$ & $6 \%$ & $0 \%$ & $4 \%$ \\
\hline $26-30 \mathrm{~cm}^{3}$ & $18 \%$ & $0 \%$ & $0 \%$ \\
\hline $31-35 \mathrm{~cm}^{3}$ & $6 \%$ & $0 \%$ & $0 \%$ \\
\hline Initial treatment duration (min) & $348($ SD 115) & $20\left(\mathrm{SD}^{\mathrm{b}}\right)$ & $60(\mathrm{SD} 26)$ \\
\hline Inpatient hospital days (days) & $11.3(\mathrm{SD} 5.8)$ & $1.0\left(\mathrm{SD}^{\mathrm{b}}\right)$ & $1.0\left(\mathrm{SD}^{\mathrm{b}}\right)$ \\
\hline
\end{tabular}


Hereinafter, values for the LINAC radiosurgery group are presented using equipment costs per fraction, with values using equipment costs per treatment in parenthesis.

The microcosting study

An overview of initial treatment costs per group, using equipment costs per fraction, is given in Table 4. Initial treatment costs were $€ 12,288$ for microsurgery, $€ 1,547$ $(€ 2,198)$ for LINAC radiosurgery, and $€ 2,412$ for gamma knife radiosurgery. There were no indications that the cost variation within and between treatment groups was related to any of the baseline characteristics. The higher costs for microsurgery were predominantly due to inpatient stay $(€ 5,321)$ and indirect costs $(€ 4,350)$.

The share of inpatient stay in total treatment costs was $43 \%$ in microsurgery, 25\% (18\%) in LINAC radiosurgery, and $16 \%$ gamma knife radiosurgery. Microsurgery patients were admitted for an average of 11.3 (SD 5.8) inpatient days. For most patients, microsurgery was performed on day 2 or 3 of admission. However, one patient underwent surgery on day 4 and one patient on day 6 of admission. Sixty-one percent of the microsurgery patients were admitted to the intensive care unit for an average of 1.0 day. LINAC and gamma knife radiosurgery are outpatient procedures. Therefore, costs for inpatient stay were over ten times higher in microsurgery than in the other two groups ( $€ 5,321$ versus $€ 386)$. Substantial cost variation was found in inpatient stay costs obtained for individual microsurgery patients (range $€ 2,318-11,201$ ).

The share of labor in total treatment costs was $15 \%$ for microsurgery, $14 \%(10 \%)$ for LINAC radiosurgery, and $10 \%$ for gamma knife radiosurgery. Labor was also an order of magnitude more expensive in microsurgery compared with the other two groups ( $€ 1,901$ compared with $€ 211$ and $€ 246)$. This was reflected by resource use of medical specialists, which amounted to $610 \mathrm{~min}$ in microsurgery (neurosurgeons and anesthesiologists), $70 \mathrm{~min}$ in LINAC radiosurgery, and $100 \mathrm{~min}$ in gamma knife radiosurgery (neurosurgeons and radiation oncologists). Besides, the microsurgical treatment required the involvement of residents (580 min), operation assistants (600 $\mathrm{min})$, and nurses (900 min), whereas the radiosurgical treatments only required radiation technicians (180 min for LINAC radiosurgery, $161 \mathrm{~min}$ for gamma knife radiosurgery) and physicists (11 min for LINAC radiosurgery, $15 \mathrm{~min}$ for gamma knife radiosurgery).

Accounting for $40 \%$ of total treatment costs, equipment was a relatively important cost driver in gamma knife radiosurgery. The replacement cost of the gamma knife $(€ 3,000,000)$ resulted in an annuity of $€ 369,873$. With maintenance costs of $€ 160,000$ per year and an average of 550 fractions or treatments per year, equipment costs per fraction or treatment were estimated to be $€ 963$. Similar
Table 4 Initial treatment costs of microsurgery, LINAC radiosurgery, and gamma knife radiosurgery (using equipment costs per fraction) (Euro 2007)

\begin{tabular}{|c|c|c|c|}
\hline & $\begin{array}{l}\text { Microsurgery } \\
(n=18)\end{array}$ & $\begin{array}{l}\text { LINAC } \\
\text { radiosurgery } \\
(n=15)\end{array}$ & $\begin{array}{l}\text { Gamma knife } \\
\text { radiosurgery } \\
(n=26)\end{array}$ \\
\hline \multicolumn{4}{|l|}{ Diagnostic procedures } \\
\hline $\begin{array}{l}\text { Medical imaging } \\
\text { services }\end{array}$ & 289 & 351 & 280 \\
\hline Laboratory services & 231 & 0 & 0 \\
\hline \multicolumn{4}{|l|}{ Consumables } \\
\hline Medications & 134 & 1 & 5 \\
\hline Disposables & 62 & 18 & 18 \\
\hline \multicolumn{4}{|l|}{ Inpatient stay } \\
\hline Normal ward & 4,142 & 386 & 386 \\
\hline Intensive care unit & 1,180 & 0 & 0 \\
\hline \multicolumn{4}{|l|}{ Labor } \\
\hline Medical specialist & 888 & 102 & 146 \\
\hline Resident & 328 & 0 & 0 \\
\hline Physicist & 0 & 8 & 11 \\
\hline Radiation technician & 0 & 101 & 90 \\
\hline Operation assistant & 297 & 0 & 0 \\
\hline Nurse & 388 & 0 & 0 \\
\hline Equipment & 0 & 50 & 963 \\
\hline Overheads and capital & 4,350 & 530 & 513 \\
\hline Initial treatment costs & 12,288 & 1,547 & 2,412 \\
\hline
\end{tabular}


calculations for the LINAC resulted in equipment costs of $€ 50$ per fraction and $€ 701$ per treatment.

The share of diagnostic procedures in initial treatment costs ranged from $4 \%$ in microsurgery to $23 \%$ (16\%) in LINAC radiosurgery. However, absolute costs of medical imaging services were comparable between treatment groups. At least one MRI or computed tomography was performed for each individual patient. Laboratory services were only carried out in microsurgery patients (€231; SD €139).

Costs for consumables accounted for less than $2 \%$ of initial treatment costs in all treatment groups. Phenytoin and nadroparine were among the most costly medications administered in microsurgery, and lidocaine and alfentanil in gamma knife radiosurgery patients. Another costly medication was dexamethasone, which was administered in all groups on indication.

The proportion of overheads and capital in the initial treatment costs was $35 \%$ for microsurgery, 34\% (24\%) for LINAC radiosurgery, and $21 \%$ for gamma knife radiosurgery.

Follow-up costs

A questionnaire was completed by each of the recruited patients. For the microsurgery group, 22\% (4/28) of the questionnaires were returned at 4 weeks, $44 \%(8 / 18)$ at 26 weeks, and $34 \%(6 / 18)$ at 52 weeks after treatment. These percentages amounted to $20 \%(3 / 15), 40 \%(6 / 15)$, and $40 \%(6 / 15)$ for the LINAC radiosurgery and $42 \%$ $(11 / 26), 31 \%(8 / 26)$, and $27 \%(7 / 26)$ for the gamma knife radiosurgery group.
The fractions of patients visiting any medical specialist were $61 \%$ for microsurgery, $20 \%$ for LINAC radiosurgery, and $38 \%$ for gamma knife radiosurgery (Pearson chi-square test: $P=0.054)$. For patients visiting the medical specialist, the annual number of visits amounted to 3,6 , and 4 , respectively (ANOVA test: $P=0.565$ ).

The fractions of patients visiting the physiotherapist were $28 \%$ for microsurgery, $40 \%$ for LINAC radiosurgery, and $19 \%$ for gamma knife radiosurgery (Pearson chi-square test: $P=0.366$ ). Two LINAC radiosurgery patients, with relatively low current health state at baseline, each received eight physiotherapist visits during the 4-week recall period. These visits were included in the calculations, but not linearly interpolated. As a result, the annual number of physiotherapist visits for patients visiting the physiotherapist amounted to nine for microsurgery, six (instead of ten) for LINAC radiosurgery, and six for gamma knife radiosurgery (ANOVA test: $P=0.848$ ).

Statistical differences were found neither in the fractions of patients visiting the general practitioner, social worker, and company physician nor in the corresponding numbers of visits per year. Resource use of medical imaging was identical in the three groups according to the established protocol. None of the patients were admitted for inpatient stay. Medications were used by about $72 \%$ of the patients in microsurgery and $73 \%$ and $65 \%$ in LINAC and gamma knife radiosurgery, respectively. In each treatment group, about one out of six patients made use of a medical aid. A summary of follow-up costs (4 weeks per measurement time point) is given in Table 5 .

Table 5 Follow-up costs (4 weeks per measurement time point) (Euro 2007) (median)

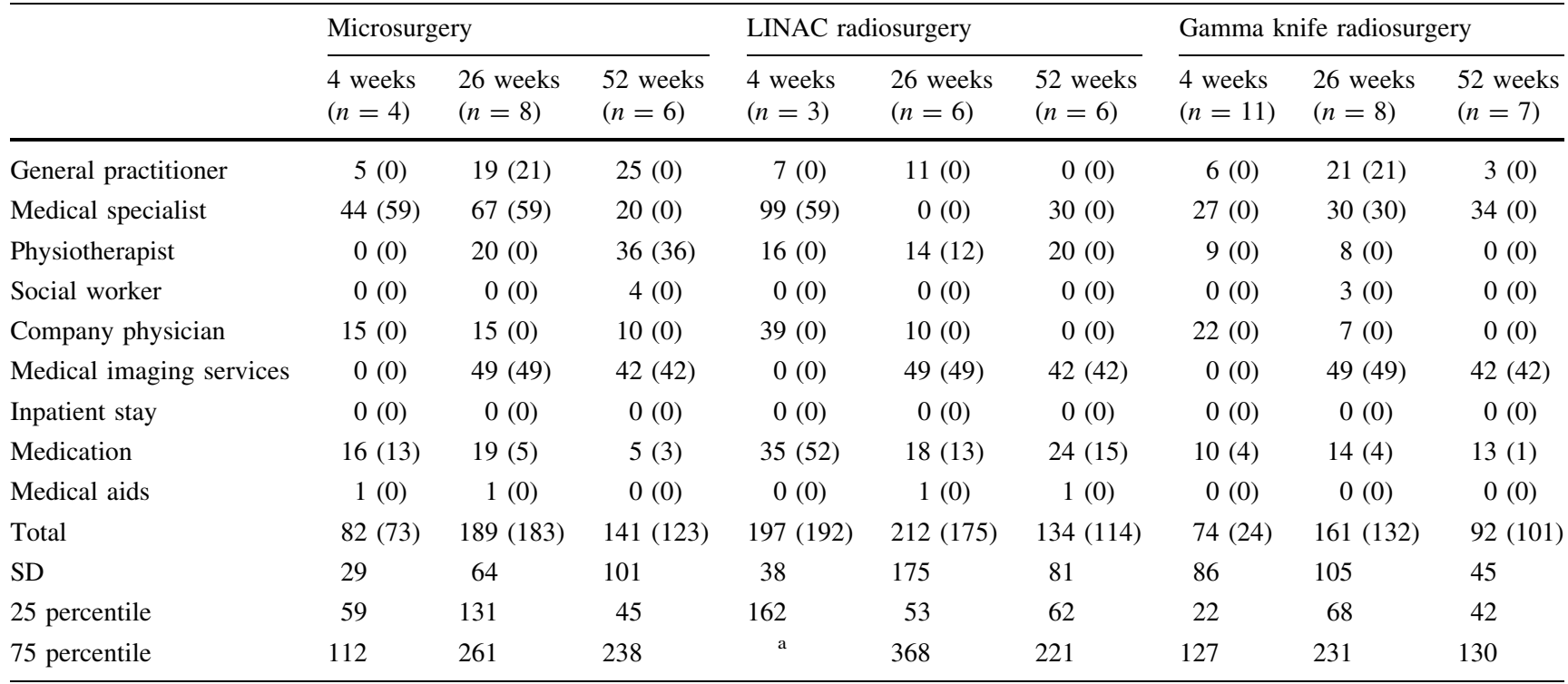

SD standard deviation

${ }^{\text {a }}$ Not available 
Table 6 Annual total costs of microsurgery, LINAC radiosurgery, and gamma knife radiosurgery (using equipment costs per fraction) (Euro 2007)

\begin{tabular}{lrrrl}
\hline & Microsurgery & $\begin{array}{l}\text { LINAC } \\
\text { Radiosurgery }\end{array}$ & $\begin{array}{l}\text { Gamma knife } \\
\text { Radiosurgery }\end{array}$ & $\begin{array}{l}\text { Kruskal-Wallis } \\
\text { Asymp. sig. }\end{array}$ \\
\hline Initial treatment costs & 12,288 & 1,547 & 2,412 & \\
Relative to microsurgery & 100 & 13 & 20 & 0.120 \\
Follow-up costs & 2,041 & 1,514 & 1,553 & 0.212 \\
General practitioner & 270 & 66 & 143 & 0.072 \\
Medical specialist & 539 & 291 & 410 & 0.429 \\
Physiotherapist & 344 & 222 & 207 & 0.675 \\
Social worker & 26 & 0 & 17 & 0.826 \\
Company physician & 160 & 94 & 62 & 0.331 \\
Medical imaging services & 540 & 540 & 540 & 1.000 \\
Inpatient stay & 0 & 0 & 0 & 0.308 \\
Medication & 156 & 290 & 172 & 0.582 \\
Medical aids & 6 & 11 & 3 & 0.000 \\
Relative to microsurgery & 100 & 74 & 76 & \\
Total costs & 14,329 & 3,060 & 3,966 & 28 \\
Relative to microsurgery & 100 & 21 & & \\
\hline
\end{tabular}

Annual follow-up costs for treatment-related and treatment-unrelated resource use consumption were $€ 2,041$ for microsurgery, $€ 1,514$ for LINAC radiosurgery, and $€ 1,553$ for gamma knife radiosurgery (Kruskal-Wallis test: $P=$ 0.120).

Annual total costs, using equipment costs per fraction, are presented in Table 6 and amounted to $€ 14,329$ for microsurgery, $€ 3,060(€ 3,711)$ for LINAC radiosurgery, and $€ 3,966$ for gamma knife radiosurgery (Kruskal-Wallis test: $P<0.001)$. Annual total costs of LINAC and gamma knife radiosurgery were not significantly different $(P=0.096)$.

\section{Discussion}

This study is the first to compare total costs of alternative procedures in the treatment of meningioma patients. With initial treatment cost of $€ 12,288$, microsurgery was the most expensive treatment option. Most important cost drivers were inpatient stay (43\%), indirect costs (35\%), and labor $(15 \%)$. This finding is in agreement with the study of Wellis et al. [8], who found initial treatment costs of microsurgery in patients harboring arteriovenous malformation, acoustic neuromas, meningiomas or brain metastasis potentially amenable to radiosurgery (diameter $<0.3 \mathrm{~cm}$ ) to be $€ 12,979$ in Germany (adjusted to 2007). In their study, inpatient stay accounted for $33 \%$, indirect costs for $39 \%$, and labor for $13 \%$ of initial treatment costs [8]. Banerjee et al. [6] determined initial treatment costs for vestibular schwannoma patients in the USA to be €22,332. However, they used general service charges rather than actual costs, which may make comparison misleading [6].
The study of Cho et al. found treatment costs for cranial base tumors (diameter $<3.0 \mathrm{~cm}$ ) to be $€ 4,628$ (adjusted to 2007) in Taiwan [12].

The two alternative methods for calculation of equipment costs were found to substantially impact our results. Initial treatment costs for gamma knife radiosurgery were $€ 866$ more expensive using equipment costs per fraction and $€ 262$ more expensive using equipment costs per treatment compared with LINAC radiosurgery. Even though the replacement values of the LINAC and gamma knife are of the same magnitude $(€ 2,500,000$ versus $€ 3,000,000$; Table 2), the average number of LINAC fractions per year was about 9,200 compared with 550 for the gamma knife. The results of our cost analysis suggest that LINAC and gamma knife radiosurgery are equally expensive when equipment is valued per treatment. This finding confirms the results of Griffiths et al. [10], who compared the equipment costs of the LINAC and gamma knife in Australia. Griffiths et al. estimated gamma knife radiosurgery to cost up to $€ 1,057$ more per fraction and to $€ 132$ more per treatment over LINAC radiosurgery (adjusted to 2007) [10].

Our results further imply that follow-up costs in the first year after initial treatment affected the relative costs of the alternative treatment options. Initial treatment costs for microsurgery, LINAC radiosurgery, and gamma knife radiosurgery correspond to the relative ratio 100:13(18):20. However, this relative ratio decreased to 100:21(26):28 when follow-up costs were included (Table 6). The latter finding is not in agreement with the results of Banerjee et al. [6]. With follow-up costs in the first year after initial treatment of approximately $€ 5,200$ for microsurgery 
patients and $€ 900$ for gamma knife radiosurgery patients (adjusted to 2007), they observed an increased relative ratio when follow-up costs were added [6]. Because Banerjee et al. [6] did not report resource use of individual cost components, it is unclear which factors led to their different conclusion.

One limitation of our study was the inclusion of resource use which was unrelated to meningioma treatment in the follow-up costs. As LINAC radiosurgery patients reported a lower current health state compared with patients in the other two treatment groups, follow-up costs of LINAC radiosurgery patients may have been relatively overestimated compared with the situation in which only resource use related to meningioma treatment would be considered. For example, $73 \%$ of medication costs were not related or possibly but not definitely related to meningioma treatment for the LINAC radiosurgery group. These percentages amounted to $67 \%$ in the microsurgery group and $68 \%$ in the gamma knife radiosurgery group. Medications directly related to meningioma treatment involved dexamethasone (often in combination with proton pump inhibitor or histamine H2-receptor antagonist) and anticonvulsants. Costs for medical aids were exclusively related to meningioma treatment for all three patient groups. Unfortunately it was not possible to assess differences between resource use related and unrelated to meningioma treatment for the other cost components (visits to healthcare providers, medical imaging services, and inpatient stay).

Our inclusion criteria restricted the recruitment of a larger number of patients. To be able to make a truthful comparison between microsurgery, LINAC radiosurgery, and gamma knife radiosurgery patients, only patients with radiologically confirmed benign (WHO grade I) meningioma with diameter $\leq 3.0 \mathrm{~cm}$ were recruited. However, at the Erasmus MC University Medical Center, microsurgery and LINAC radiosurgery were not commonly performed in meningioma with diameter $\leq 3.0 \mathrm{~cm}$. Besides, the fact that microsurgery patients were only included when they could have been treated with radiosurgery instead limited recruitment of microsurgery patients.

Consequently, our study included only a small sample of patients, especially for the calculation of follow-up costs in which the microcosting patient samples were further divided into three subgroups to be able to detect follow-up costs at 4,26 , and 52 weeks. The small sample of patients prevented us from further omitting patients due to differences in baseline characteristics. For example, the shares of tuberculum sellae and sphenoid ridge patients were (not significantly) higher and those of cavernous sinus and cerebellopontine angle patients (not significantly) lower in microsurgery. In addition, follow-up costs for the time between measurement periods (week 5-22 and week 27-48) were established through linear interpolation, which may have affected the results. Our results may therefore not be representative of clinical treatment patterns in The Netherlands. However, there were no indications that the cost variation within and between treatment groups was related to any of the baseline characteristics. Moreover, costing studies which assess followup costs are scarce, and thus we believe that our study provides valuable insight into the relative costs of alternative procedures for treatment of meningioma.

To determine the uncertainty of the obtained cost estimates, one-way sensitivity analyses were carried out by individually varying each unit cost value between $50 \%$ and $150 \%$. Although total costs changed $25-43 \%$ when the initial treatment costs were varied, the relative ratio for microsurgery, LINAC radiosurgery, and gamma knife radiosurgery did not change substantially. The influence of this variation appeared to be rather modest for the other cost components. Total costs changed 1-2\% when unit costs of visits to the general practitioner were altered (follow-up costs: $2-7 \%$ ). This change amounted to $2-5 \%$ for visits to the medical specialist (follow-up costs: $10-13 \%$ ), 1-4\% for visits to the physiotherapist (follow-up costs: 7-8\%), 2-9\% for medical imaging services (follow-up costs: 13-18\%), and $1-5 \%$ for medications (follow-up costs: 4-10\%).

Meningioma may be treated with procedures other than microsurgery, LINAC radiosurgery, and gamma knife radiosurgery. Yano and Kuratsu suggested that conservative treatment with close monitoring may be the best therapeutic strategy in asymptomatic meningioma patients to avoid incidence of surgery-related morbidity [11]. Furthermore, combination therapy of microsurgery and radiosurgery may be beneficial [7, 24]. When meningioma does not respond favorably to microsurgery and/or radiosurgery, alternative procedures may include chemotherapy, immunotherapy, hormone therapy, gene therapy, and/or toxins [5]. However, cost information on these treatment options is not yet available.

Earlier studies by others suggested radiosurgery to be more cost-effective than microsurgery [6, 12, 13]. Rutigliano et al. concluded that radiosurgery resulted in favorable costs per life year compared with surgical resection in solitary metastatic brain tumors [19]. Myrseth et al. observed significantly favorable posttreatment facial nerve function, hearing, complication rates, and quality of life for gamma knife radiosurgery over microsurgery in unilateral vestibular schwannoma patients [13]. Unfortunately, our study did not weigh the costs of meningioma treatment against clinical outcome measures, which prevented us from drawing conclusions regarding the relative cost-effectiveness of the three investigated treatments. To be able to better assist clinical decision-making for meningioma patients, future studies should determine the cost-effectiveness of microsurgery, LINAC radiosurgery, 
and gamma knife radiosurgery by including clinical outcome measures and quality of life, preferably over a longer timeframe.

Furthermore, future studies should consider productivity costs due to absence from work and reduced efficiency at paid and unpaid work. As our study was conducted from the healthcare providers' perspective, it disregarded productivity costs. However, as radiosurgery is a minimally invasive outpatient procedure, a productivity cost reduction over microsurgery may be expected. Therefore, productivity costs could significantly affect the cost-effectiveness of microsurgery, LINAC radiosurgery, and gamma knife radiosurgery in meningioma patients $[8,12,20]$.

Acknowledgements The authors would like to thank the employees of the medical and financial departments of the Erasmus MC University Medical Center in Rotterdam and the St. Elisabeth Hospital in Tilburg who provided resource use and cost data for the study. The authors are grateful to Arnold Dijkstra, Peter Levendag, Susan Moederscheim, Connie de Pan, and Saskia Schawo for their contribution to this study. This study was supported by Elekta BV.

Open Access This article is distributed under the terms of the Creative Commons Attribution Noncommercial License which permits any noncommercial use, distribution, and reproduction in any medium, provided the original author(s) and source are credited.

\section{References}

1. Riemenschneider MJ, Perry A, Reifenberger G (2006) Histological classification and molecular genetics of meningiomas. Lancet Neurol 5(12):1045-1054

2. Rockhill J, Mrugala M, Chamberlain MC (2007) Intracranial meningiomas: an overview of diagnosis and treatment. Neurosurg Focus 23(4):E1

3. Campbell BA, Jhamb A, Maguire JA, Toyota B, Ma R (2009) Meningiomas in 2009: controversies and future challenges. Am J Clin Oncol 32(1):73-85

4. Louis DN, Ohgaki H, Wiestler OD et al (2007) The 2007 WHO classification of tumours of the central nervous system. Acta Neuropathol 114(2):97-109

5. D'Ambrosio AL, Bruce JN (2003) Treatment of meningioma: an update. Curr Neurol Neurosci Rep 3(3):206-214

6. Banerjee R, Moriarty JP, Foote RL, Pollock BE (2008) Comparison of the surgical and follow-up costs associated with microsurgical resection and stereotactic radiosurgery for vestibular schwannoma. J Neurosurg 108(6):1220-1224

7. Bennett CL, Tigue CC, Fitzner KA (2007) The economics of brain metastases. Cancer Treat Res 136:23-29

8. Wellis G, Nagel R, Vollmar C, Steiger HJ (2003) Direct costs of microsurgical management of radiosurgically amenable intracranial pathology in Germany: an analysis of meningiomas, acoustic neuromas, metastases and arteriovenous malformations of less than $3 \mathrm{~cm}$ in diameter. Acta Neurochir (Wien) 145(4): 249-255

9. Elia AE, Shih HA, Loeffler JS (2007) Stereotactic radiation treatment for benign meningiomas. Neurosurg Focus 23(4):E5

10. Griffiths A, Marinovich L, Barton MB, Lord SJ (2007) Cost analysis of Gamma Knife stereotactic radiosurgery. Int J Technol Assess Health Care 23(4):488-494

11. Yano S, Kuratsu J (2006) Indications for surgery in patients with asymptomatic meningiomas based on an extensive experience. J Neurosurg 105(4):538-543

12. Cho DY, Tsao M, Lee WY, Chang CS (2006) Socioeconomic costs of open surgery and gamma knife radiosurgery for benign cranial base tumors. Neurosurgery 58(5):866-873 discussion 866-873

13. Myrseth E, Moller P, Pedersen PH, Vassbotn FS, Wentzel-Larsen T, Lund-Johansen M (2005) Vestibular schwannomas: clinical results and quality of life after microsurgery or gamma knife radiosurgery. Neurosurgery 56(5):927-935 discussion 927-935

14. Perks JR, St George EJ, El Hamri K, Blackburn P, Plowman PN (2003) Stereotactic radiosurgery XVI: isodosimetric comparison of photon stereotactic radiosurgery techniques (gamma knife vs. micromultileaf collimator linear accelerator) for acoustic neuroma-and potential clinical importance. Int J Radiat Oncol Biol Phys 57(5):1450-1459

15. Mehta M, Noyes W, Craig B et al (1997) A cost-effectiveness and cost-utility analysis of radiosurgery vs. resection for single-brain metastases. Int J Radiat Oncol Biol Phys 39(2):445-454

16. Porter PJ, Shin AY, Detsky AS, Lefaive L, Wallace MC (1997) Surgery versus stereotactic radiosurgery for small, operable cerebral arteriovenous malformations: a clinical and cost comparison. Neurosurgery 41(4):757-764 discussion 764-766

17. Ott K (1996) A comparison of craniotomy and Gamma Knife charges in a community-based Gamma Knife Center. Stereotact Funct Neurosurg 66 Suppl 1:357-364

18. van Roijen L, Nijs HG, Avezaat CJ et al (1997) Costs and effects of microsurgery versus radiosurgery in treating acoustic neuroma. Acta Neurochir (Wien) 139(10):942-948

19. Rutigliano MJ, Lunsford LD, Kondziolka D, Strauss MJ, Khanna V, Green M (1995) The cost effectiveness of stereotactic radiosurgery versus surgical resection in the treatment of solitary metastatic brain tumors. Neurosurgery 37(3):445-453 discussion 453-5

20. Drummond MF, Sculpher MJ, Torrance GW, O'Brien BJ, Stoddart GL (2005) Methods for the economic evaluation of health care programmes. Oxford University Press, New York

21. Tan SS, Rutten FF, van Ineveld BM, Redekop WK, Hakkaart-van Roijen L (2009) Comparing methodologies for the cost estimation of hospital services. Eur J Health Econ 10(1):39-45

22. Centraal Bureau voor de Statistiek, Ministerie voor Volksgezondheid Welzijn en Sport (2007) www.statline.nl

23. Oostenbrink JB, Koopmanschap MA, Rutten FF (2002) Standardisation of costs: the Dutch Manual for Costing in economic evaluations. Pharmacoeconomics 20(7):443-454

24. Black PM, Villavicencio AT, Rhouddou C, Loeffler JS (2001) Aggressive surgery and focal radiation in the management of meningiomas of the skull base: preservation of function with maintenance of local control. Acta Neurochir (Wien) 143(6): $555-562$ 